\title{
LETTER \\ Salient Region Detection Based on Color Uniqueness and Color Spatial Distribution
}

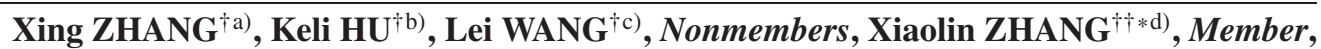 \\ and Yingguan WANG ${ }^{\dagger}$ ) , Nonmember
}

\begin{abstract}
SUMMARY In this study, we address the problem of salient region detection. Recently, saliency detection with contrast based approaches has shown to give promising results. However, different individual features exhibit different performance. In this paper, we show that the combination of color uniqueness and color spatial distribution is an effective way to detect saliency. A Color Adaptive Thresholding Watershed Fusion Segmentation (CAT-WFS) method is first given to retain boundary information and delete unnecessary details. Based on the segmentation, color uniqueness and color spatial distribution are defined separately. The color uniqueness denotes the color rareness of salient object, while the color spatial distribution represents the color attribute of the background. Aiming at highlighting the salient object and downplaying the background, we combine the two characters to generate the final saliency map. Experimental results demonstrate that the proposed algorithm outperforms existing salient object detection methods.
\end{abstract}

key words: visual attention, saliency map, color uniqueness, color spatial distribution

\section{Introduction}

The identification of salient regions that capture our visual attention is a cross-disciplinary problem. Visual attention achieved in computational system is a combination of the insights from neuroscience, biology, computer vision, and other fields. The estimation of salient regions is desirable for the ability to allocate computational resources for image analysis in advance. Recent studies have shown that bottomup stimuli-driven attention models [1], [2] could be effectively applied to many application scenarios, which contain image segmentation, resizing, and object recognition.

Results from recent research [3] indicate that contrast based salient detection is an effective model for low-level

Manuscript received January 7, 2014.

Manuscript revised February 28, 2014.

${ }^{\dagger}$ The authors are with the Internet of Things System Technology Laboratory, Shanghai Institute of Microsystem and Information Technology, Chinese Academy of Sciences, Shanghai, 200050, P.R. China.

${ }^{\dagger \dagger}$ The author is with the Precision and Intelligence Laboratory Zhang Group, Tokyo Institute of Technology, Tokyo, 152-8550 Japan.

*Presently, with the Internet of Things System Technology Laboratory, Shanghai Institute of Microsystem and Information Technology, Chinese Academy of Sciences, Shanghai, 200050, P.R. China.

a) E-mail: zhangxingfelix @gmail.com

b)E-mail: ancimoon@gmail.com

c)E-mail: wanglei@gmail.com

d) E-mail: xlzhang@mail.sim.ac.cn

e) E-mail: wyg@mail.sim.ac.cn

DOI: 10.1587/transinf.E97.D.1933 visual saliency. However, the definition of contrast is quite different, including edges, gradient, spatial frequencies, histograms, multi-scale descriptors, or a combination of certain features. Different individual features yield different performance attributes. This work focuses on the key attribute of color. Ideally, colors belonging to the background will be distributed over the entire image exhibiting a high spatial variance, whereas foreground objects are generally more compact [4].

In this work, we concentrate on the combined results of salient region and background detection both by using contrast based approach, in order to highlight the salient regions and downplay the background regions. The prerequisite of the contrast is segmentation, which plays an important role in the final results. Here, we propose a Color Adaptive Thresholding Watershed Fusion Segmentation (CAT-WFS) algorithm to retain more boundary information and merge regions with low color difference. The segmentation process also suppresses the undesirable details.

To detect the salient regions, we propose an algorithm to calculate the color uniqueness, which is a key attribute to reflect the visual rareness of saliency. The color uniqueness is defined by region color difference and has no relationship with distance information. To determine the background, a color spatial distribution method is given, with the assumption that the color of low salient areas should be widely distributed. The final saliency map is generated by the product of color uniqueness and color spatial distribution. The method is called color uniqueness and distribution method (CUD), which is shown in Fig. 1.

The remainder of this paper is organized as follows. In Sect. 2, the proposed salient region detection method based

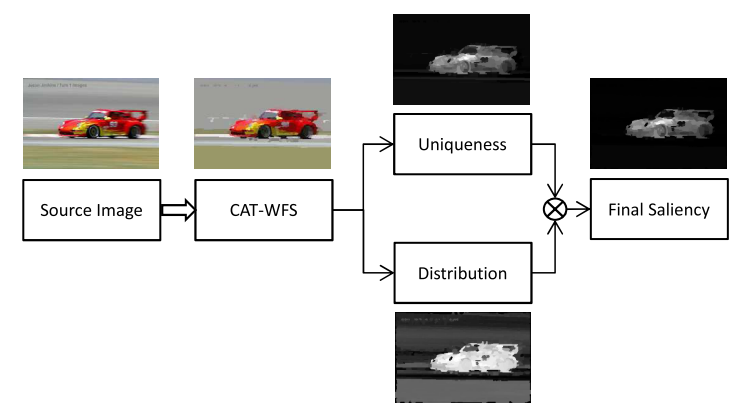

Fig. 1 The exemplary process of color uniqueness and distribution method (CUD) saliency region detection. 
on color uniqueness and color spatial distribution algorithm is presented. Experimental evaluations and discussions are presented in Sect. 3, and Sect. 4 is the conclusion.

\section{Salient Region Detection Algorithm}

In this section, we describe the details of our method. As motivated above, we propose an algorithm that segments the image into regions in a fusion way. The corresponding color uniqueness and color spatial distribution algorithms are discussed in detail. The final fusion of the two key elements is provided in the last.

\subsection{Region Fusion Segmentation}

Watershed algorithm began with the work of Maxwell, that is how to divide a landscape into regions such as hills and dales [5]. Since then, algorithms to compute watershed were developed in the field of topography to handle digital elevation model [6]. To retain the boundary information, we adopt float-point Gaussian watershed segmentation to over segment the input image. The parameter $\sigma$ of Gaussian filter determines the region number of watershed segmentations. The larger $\sigma$ is, the fewer the segments are. In our approach, we adopt $\sigma=1.0$ to over segment the original image.

The original image is decomposed into regions with unnecessary details. To abstract unnecessary details and preserve relevant structure with enough boundary information, we introduce Color Adaptive Thresholding Watershed Fusion Segmentation (CAT-WFS). After the float-point Gaussian watershed segmentation, the image I is segmented into regions $r_{i}, i=1,2, \ldots, M$. The average color of region $r_{i}$ is $C\left(r_{i}\right)$. If two adjacent regions $r_{i}$ and $r_{j}$ meet the requirement defined by Eq. (1), the two regions are fused into one. Equation (1) is defined as:

$$
\left|C\left(r_{i}\right)-C\left(r_{j}\right)\right| \leq T_{a}
$$

where, $T_{a}$ is the adaptive fusion threshold. The average color $C\left(r_{i}\right)$ is calculated in $\mathrm{L}^{*} \mathrm{a} * \mathrm{~b}$ color space.

In our approach, the fusion threshold is image mean color difference dependent, instead of constant threshold for each image. The adaptive threshold $\left(T_{a}\right)$ is determined by:

$$
T_{a}=\frac{1}{M \times k} \sum_{x=0}^{W-1} \sum_{y=0}^{H-1}\left(I(x, y)-I_{a}\right)
$$

where $W$ and $H$ are the width and height of the over segmented image in pixels, respectively, and $I(x, y)$ is the color value of the pixel at position $(x, y), I_{a}$ is the average color of the image. $M$ is the region number of segmentation, and $k$ is a parameter to control $T_{a}$. In our implementation, we use $k=4$ to do the region fusion.

\subsection{Color Uniqueness}

The color uniqueness is defined as the global contrast of region mean color, in order to represent the rarity of salient region. As to a single region $r_{k}$, the corresponding color uniqueness $U\left(r_{k}\right)$ is calculated by:

$$
U\left(r_{k}\right)=\sum_{r_{k} \neq r_{i}} \omega\left(r_{i}\right) D_{r}\left(r_{k}, r_{i}\right)
$$

where $\omega\left(r_{i}\right)$ is the weighted value of region $r_{i}$. In our work, $\omega\left(r_{i}\right)$ is presented as the point number of region $r_{i} . D_{r}(\cdot, \cdot)$ denotes the color difference of two regions, which is defined as:

$$
D_{r}\left(r_{k}, r_{j}\right)=\left\|I_{k}-I_{j}\right\|
$$

where $I_{k}$ is the mean color of region $r_{i}$. Notice that the color difference is measured in $\mathrm{L}^{*} \mathrm{a} * \mathrm{~b}$ color space. After the calculation process, the color uniqueness $U\left(r_{k}\right)$ is normalized into $[0,1]$, which is denoted by $U_{0}\left(r_{k}\right)$.

\subsection{Color Spatial Distribution}

Ideally, the color of salient object should be limited in a certain area, and the color belong to background should has a wide distribution [12]. Here, we define the color spatial distribution $D\left(r_{i}\right)$ of region $r_{i}$ :

$$
D\left(r_{i}\right)=\sum_{j=1}^{N}\left\|P_{j}-\mu_{i}\right\|^{2} \omega_{i j}
$$

where $\omega_{i j}$ is the similarity of region $r_{i}$ and $r_{j}$, and is defined below:

$$
\omega_{i j}=\frac{1}{z_{i}} \exp \left(-\frac{1}{2 \delta_{c}^{2}}\left\|I_{i}-I_{j}\right\|^{2}\right) \omega\left(r_{i}\right)
$$

where $\omega\left(r_{i}\right)$ still denote the point number of region $r_{i} . z_{i}$ is the normalization factor to keep $\sum_{i=1}^{N} \omega\left(r_{i}\right)=1$. In Eq. (5), $P_{j}$ is the mean position of region $r_{j}$ and $\mu_{i}$ is the weighted average position of color $I_{i}$, which is defined by:

$$
\mu_{i}=\sum_{j=1}^{N} \omega_{i j} P_{j}
$$

The parameter $\delta_{c}$ in Eq. (6) controls the sensitivity of the color spatial distribution. In this work, we adopt $\delta_{c}=6$ in all the experiments. Notice that, the color difference in Eq. (6) is also computed in $\mathrm{L}^{*} \mathrm{a} * \mathrm{~b}$ color space.

After the computation, $D\left(r_{i}\right)$ should be normalized to $[0,1]$, which we use $D_{n}\left(r_{i}\right)$ to represent. In order to make a comparison to color uniqueness, we adopt the reverse transition:

$$
D_{0}\left(r_{i}\right)=1-D_{n}\left(r_{i}\right)
$$

\subsubsection{The Final Saliency Map Generation}

With the results from Sects.2.2 and 2.3, we make a combination of color uniqueness and color spatial distribution. The final saliency value $S\left(r_{i}\right)$ of region $r_{i}$ is defined as:

$$
S\left(r_{i}\right)=U_{0}\left(r_{i}\right) \times D_{0}\left(r_{i}\right)
$$




\section{Experimental Results and Discussion}

The results of CUD are evaluated on two benchmarks. Benchmark I is provided by Achanta et al. [7], which contains 1000 images with binary ground truth. Benchmark II is provided by Li and Ngan [15], which contains $210 \mathrm{im}-$ ages with binary ground truth. We compare our work with SR [9], MZ [12], FT [7], LC [16], HC [17], RC [17], CA [4], GB [10], AC [11], IT [8], SF [14], GC [13], and CO [15]. Visual comparisons to different state-of-the-art approaches are given in Figs. 2 and 3 with images randomly selected from the two benchmarks separately.

According to [7], [17], we evaluate our method by calculating its precision and recall rate. Precision measures the pixels that are correctly assigned in percentage, and Re- call measures the salient pixels that are correctly detected in percentage. First we adopt fixed threshold in the range of $[0 \ldots 255]$ to generate corresponding binary saliency maps. Each binary saliency map is assigned to a precision and recall value with comparison to the ground truth mask. The resulting precision and recall curve is shown in Fig. 4 (a).

In the second evaluation, we adopt the image depend threshold proposed by [7], defined as twice the mean saliency of the image:

$$
T_{a}=\frac{2}{W \times H} \sum_{x=1}^{W} \sum_{y=1}^{H} S(x, y)
$$

where $W$ and $H$ are the width and height of the saliency map $S$ and $S(x, y)$ is the saliency value in saliency map $S$ within the position $(x, y)$. The weighted harmonic measure

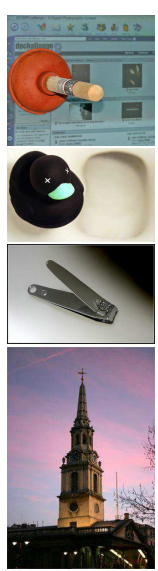

(a) IMG

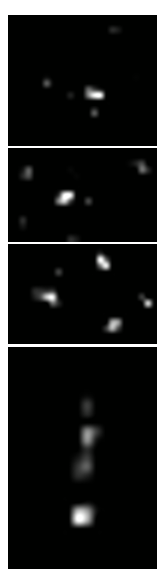

(b) IT

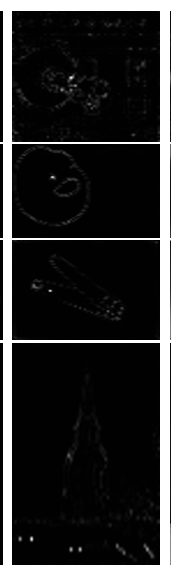

(c) SR

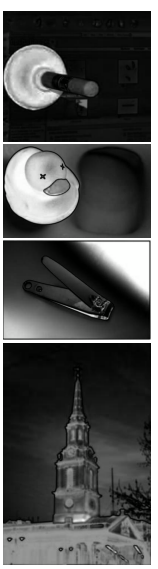

(d) FT

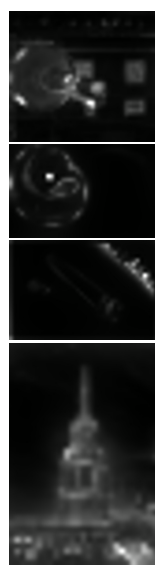

(e) GB

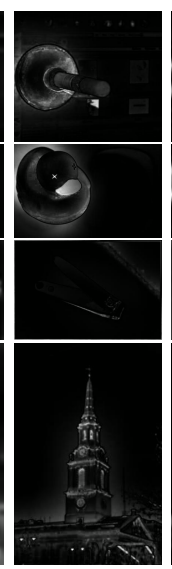

(f) $\mathrm{AC}$

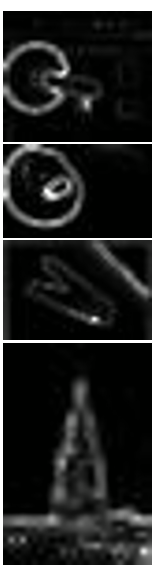

(g) MZ

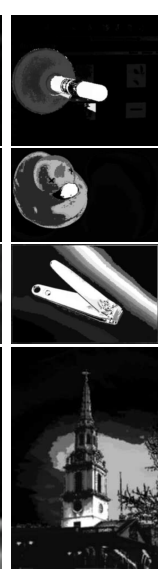

(h) GC

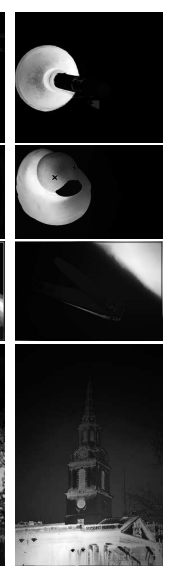

(i) $\mathrm{SF}$

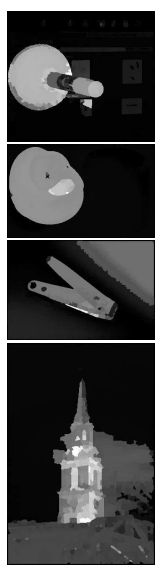

(j) CUD

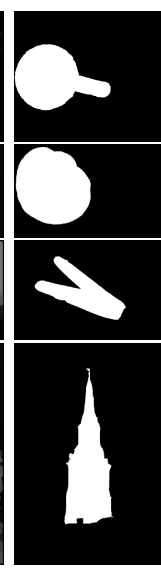

(k) GT

Fig. 2 Visual comparison of saliency maps on benchmark I[7]. (a) Original images, (b) IT [8], (c) SR [9], (d) FT [7], (e) GB [10], (f) AC [11], (g) MZ [12], (h) GC [13], (i) SF [14], (j) CUD, (k) Ground truth.

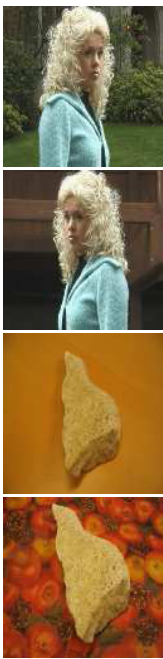

(a) IMG

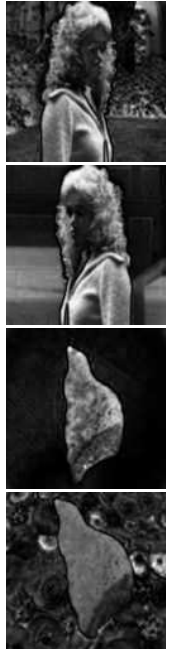

(b) FT

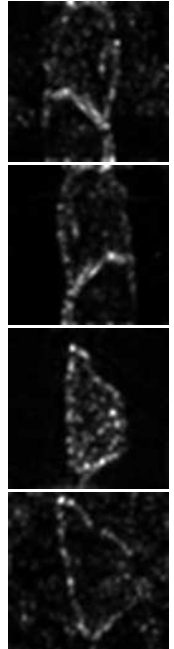

(c) SR

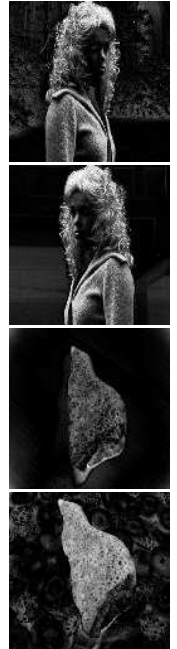

(d) LC

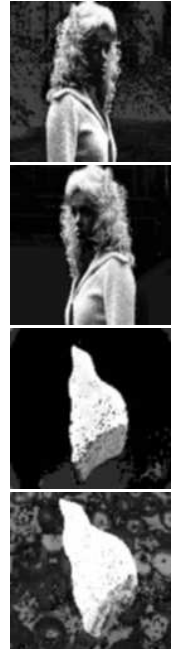

(e) $\mathrm{HC}$

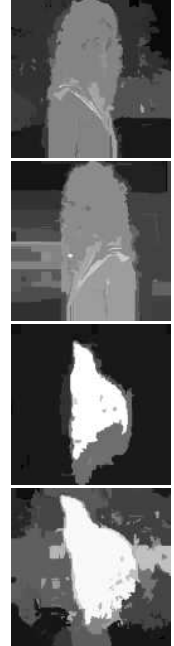

(f) RC

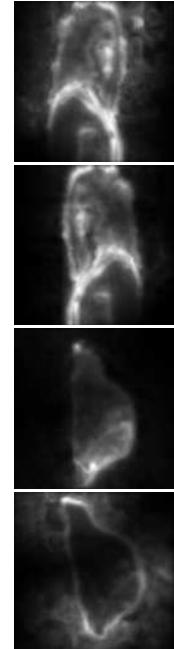

(g) $\mathrm{CA}$

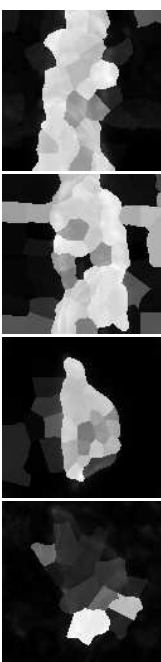

(h) $\mathrm{CO}$

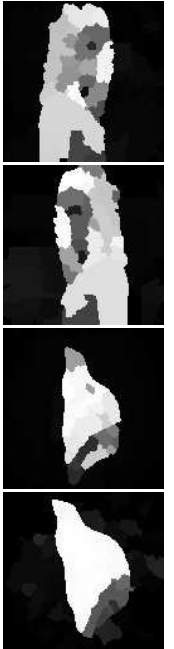

(i) CUD

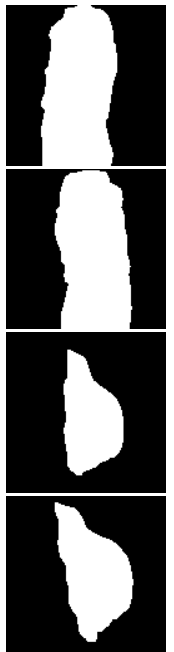

(j) GT

Fig. 3 Visual comparison of saliency maps on benchmark II [15]. (a) Original images, (b) FT [7], (c) SR [9], (d) LC [16], (e) HC [17], (f) RC [17], (g) CA [4], (h) CO [15], (i) CUD, (j) Ground truth. 

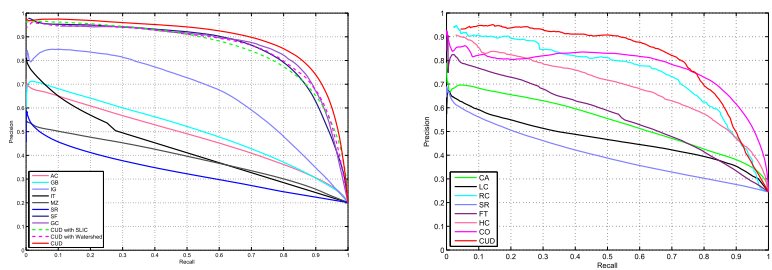

(a) Fixed Thresholding
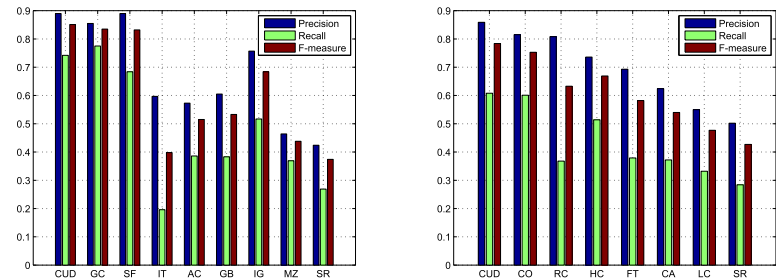

(b) Adaptive Thresholding

Fig. 4 Statistical comparison with 13 other saliency detection methods using the benchmarks provided by [7] (left) and [15] (right). (a) and (b) are average precision and recall curves by fixed/adaptive thresholding separately. Our method CUD is compared with SR [9], MZ[12], FT [7], LC [16], HC [17], RC [17], CA [4], GB [10], AC [11], IT [8], SF [14], GC [13], and CO [15].

or F-measure is also computed, which is defined as:

$$
F_{\beta}=\frac{\left(1+\beta^{2}\right) \cdot \text { Precision } \cdot \text { Recall }}{\beta^{2} \cdot \text { Precision }+ \text { Recall }}
$$

Similar to [7], [14], [17], we set $\beta^{2}=0.3$ to weigh precision more than recall. The resulting adaptive threshold precision and recall curve is shown in Fig. 4 (b).

In Fig. 4 (a), we compare CUD with other methods by fixed threshold. The left figure is measured on benchmark I [7], our method CUD has higher precision rate compared to other 8 approaches. As discussed in Sect. 2.1, a comparison between CAT-WFS and SLIC superpixels [18], watershed [6] is also given in this figure. CUD with CATWFS outperforms CUD with SLIC superpixels or watershed segmentation. The right figure in Fig. 4 (a) is measured on benchmark II [15]. Our method CUD has higher precision rate compared to other 7 approaches. Meanwhile, in Fig. 4 (b), we compare CUD with other methods by adaptive threshold. Our method CUD has the highest F-measure value on the two benchmarks, which is 0.851 and 0.784 separately.

\section{Conclusion}

In this paper, a method of salient region detection based on color uniqueness and color spatial distribution is proposed. We employ a region fusion segmentation method based on float-point watershed segmentation to generate segmentations retaining strong boundary information and fusing over segmented regions. Color uniqueness is defined to identify salient regions through the rarity of color. Color spatial distribution is determined by the color attribute of the background. The final saliency map is generated by taking the product of color uniqueness and color spatial distribution to highlight the saliency object and downplay the background. Through the precision and recall curve evaluation on two different datasets, our method CUD outperforms other stateof-the-art approaches.

\section{Acknowledgments}

This work is supported by the Plan of Action on Science and Technology Innovation, Science and Technology Commission of Shanghai Municipality (Project Number:13511507503).

\section{References}

[1] L. Itti and P.F. Baldi, "Bayesian surprise attracts human attention," Advances in Neural Information Processing Systems, pp.547-554, 2006.

[2] C. Koch and S. Ullman, Shifts in selective visual attention: Towards the underlying neural circuitry, pp.115-141, Springer, 1987.

[3] W. Einhäuser and P. König, "Does luminance-contrast contribute to a saliency map for overt visual attention?," European Journal of Neuroscience, vol.17, no.5, pp.1089-1097, 2003.

[4] S. Goferman, L. Zelnik-Manor, and A. Tal, "Context-aware saliency detection," IEEE Trans. Pattern Anal. Mach. Intell., vol.34, no.10, pp.1915-1926, 2012.

[5] J.C. Maxwell, "L. on hills and dales: To the editors of the philosophical magazine and journal," 1870.

[6] J.M. Gauch, "Image segmentation and analysis via multiscale gradient watershed hierarchies," IEEE Trans. Image Process., vol.8, no.1, pp.69-79, 1999.

[7] R. Achanta, S. Hemami, F. Estrada, and S. Süsstrunk, "Frequencytuned salient region detection," IEEE Conference on Computer Vision and Pattern Recognition, CVPR 2009, pp.1597-1604, 2009.

[8] L. Itti, C. Koch, and E. Niebur, "A model of saliency-based visual attention for rapid scene analysis," IEEE Trans. Pattern Anal. Mach. Intell., vol.20, no.11, pp.1254-1259, 1998.

[9] X. Hou and L. Zhang, "Saliency detection: A spectral residual approach," IEEE Conference on Computer Vision and Pattern Recognition, CVPR '07, pp.1-8, 2007.

[10] J. Harel, C. Koch, and P. Perona, "Graph-based visual saliency," Advances in neural information processing systems, pp.545-552, 2007.

[11] R. Achanta, F. Estrada, P. Wils, and S. Süsstrunk, Salient region detection and segmentation, pp.66-75, Springer, 2008.

[12] Y.F. Ma and H.J. Zhang, "Contrast-based image attention analysis by using fuzzy growing," Proc. Eleventh ACM International Conference on Multimedia, pp.374-381, 2003.

[13] M.M. Cheng, J. Warrell, W.Y. Lin, S. Zheng, V. Vineet, and N. Crook, "Efficient salient region detection with soft image abstraction,” IEEE ICCV, pp.1529-1536, 2013.

[14] F. Perazzi, P. Krahenbuhl, Y. Pritch, and A. Hornung, "Saliency filters: Contrast based filtering for salient region detection," 2012 IEEE Conference on Computer Vision and Pattern Recognition (CVPR), pp.733-740, 2012.

[15] H. Li and K.N. Ngan, "A co-saliency model of image pairs," IEEE Trans. Image Process., vol.20, no.12, pp.3365-3375, 2011.

[16] Y. Zhai and M. Shah, "Visual attention detection in video sequences using spatiotemporal cues," Proc. 14th Annual ACM International Conference on Multimedia, pp.815-824, 2006.

[17] M.M. Cheng, G.X. Zhang, N.J. Mitra, X. Huang, and S.M. Hu, "Global contrast based salient region detection," IEEE CVPR, pp.409-416, 2011.

[18] R. Achanta, A. Shaji, K. Smith, A. Lucchi, P. Fua, and S. Süsstrunk, "Slic superpixels," Ecole Polytechnique Fédéral de Lausssanne (EPFL), Tech. Rep., vol.2, p.3, 2010. 